\title{
TECHNIQUES FOR CLUSTERING GENE EXPRESSION DATA
}

\author{
G. Kerr *, H.J. Ruskin, M. Crane, P. Doolan \\ Biocomputation Research Lab, (Modelling and Scientific Computing Group, School \\ of Computing) and National Institute of Cellular Biotechnology, Dublin City \\ University, Dublin 9, Ireland.
}

\begin{abstract}
Many clustering techniques have been proposed for the analysis of gene expression data obtained from microarray experiments. However, choice of suitable method(s) for a given experimental dataset is not straightforward. Common approaches do not translate well and fail to take account of the data profile. This review paper surveys state of the art applications which recognise these limitations and addresses them. As such, it provides a framework for the evaluation of clustering in gene expression analyses. The nature of microarray data is discussed briefly. Selected examples are presented for clustering methods considered.
\end{abstract}

Key words: Gene Expression, Clustering, Bi-clustering, Microarray Analysis

\section{Introduction}

Searching for meaningful information patterns and dependencies in gene expression (GE) data, to provide a basis for hypothesis testing is non-trivial. An initial step is to cluster or "group" genes, with similar changes in expression. Lack of a priori knowledge means that unsupervised clustering techniques, where data are unlabeled (un-annotated), are common in GE work. These are an exploratory techniques and assume that there is an unknown mapping that assigns a group "label" to each gene, where the goal is to estimate this mapping. However, common clustering approaches do not always translate well to GE data, and may fail significantly to account for data profile.

\footnotetext{
* Biocomputation Research Lab, Modelling and Scientific Group, Dublin City University, Dublin 9, Ireland

Email address: gkerr@computing.dcu.ie (G. Kerr).
} 
Many excellent reviews of GE analysis, using clustering techniques, are available. Asyali et al. [1] provide a synopsis of class prediction and discovery (respectively, supervised pattern recognition and clustering), while Pham et al. [2] provide a comprehensive literature review of the various stages of data analysis during a microarray experiment. In a landmark paper Jain et al. [3] provide a thorough introduction to clustering, and give a taxonomy of clustering algorithms, (used in this review). Reviewing the state of the art in GE analysis is complicated by the high level of interest in the field, and the many techniques available. This review aims to evaluate modifications to currentlyused techniques which address shortcomings of conventional approaches and special properties of GE data.

GE data are typically presented as a real-valued matrix, with row objects corresponding to GE measurements over a number of experiments, and columns corresponding to the pattern of expression of all genes for a given microarray experiment. Each entry, $x_{i j}$, is the measured expression of gene $i$ in experiment $j$. Dimensionality of a gene refers to the number of its expression values recorded (number of matrix columns). A gene/gene profile $x$ is a single data item (row) consisting of $d$ measurements, $x=\left(x_{1}, x_{2}, \ldots, x_{d}\right)$. An experiment/sample $y$ is a single microarray experiment corresponding to a single column in the GE matrix, $y=\left(x_{1}, x_{2}, \ldots, x_{n}\right)^{T}$ where $n$ is the number of genes in the dataset.

Accuracy of GE data strongly depends on experimental design and minimisation of technical variation, whether due to instruments, observer or preprocessing [4]. Image corruption and/or slide impurities may led to incomplete data [5]. Many clustering algorithms require a complete matrix of input values, so imputation, (missing data estimation), techniques need to be considered before clustering. GE data is intrinsically noisy, resulting in outliers, typically managed by: (i) robust statistical estimation/testing, (when extreme values are not of primary interest), or (ii) identification, (when outlier information is of intrinsic importance, [6]. As cluster analysis is usually exploratory, lack of a priori knowledge on gene groups or their number, $K$, is common. Arbitrary selection of this number may undesirably bias the search, as pattern elements may be ill-defined unless signals are strong. Meta-data can guide choice of correct $K$, e.g. genes with common promoter sequence are likely to be expressed together and thus are likely to be placed in the same group. Methods for determining optimal number of groups, $K$, are discussed in [7] and [8].

Clustering a GE matrix can be achieved in two ways: (i) genes can form a group which show similar expression across conditions, (ii) samples can form a group which show similar expression across all genes. Both (i) and (ii) lead to global clusters, where a gene or sample is grouped across all dimensions. However, genes and samples can be clustered simultaneously, with their interrelationship represented by bi-clusters. These are defined over a subset of genes 
and a subset of samples thus capturing local structure in the dataset. This is a major strength of bi-clustering as cellular processes are understood to rely on subsets of genes, which are co-regulated and co-expressed under certain conditions and behave independently under others, [9]. Justifiably, this approach has been gaining much interest of late. For an excellent review on bi-clusters and bi-clustering techniques see [10].

Additionally, clustering can be complete or partial, where the former assigns each gene to a cluster, and the latter does not. Partial clustering tends to be more suited to GE, as the dataset often contains irrelevant genes or samples. This allows: (i) "noisy genes" to be left out, with correspondingly less impact on the outcome and (ii) genes to belong to no cluster - omitting a large number of irrelevant contributions. This is important as microarrays measure expression for the entire genome in one experiment, but genes may change expression independent of the experimental condition (e.g. due to stage in the cell cycle). Forced inclusion, (as demanded by complete clustering), in well-defined but inappropriate groups, may impact final structure found for the data. Partial clustering thus avoids the situation where an interesting sub-group in a cluster is obscured through forcing membership of unrelated genes.

Finally, clustering can be categorised as exclusive (hard), or overlapping. Exclusive clustering requires each gene to belong to a single cluster, whereas overlapping clusters permit genes simultaneously to be members of numerous clusters. An additional qualification is crisp and fuzzy membership. Crisp membership is boolean - either the gene belongs to a group or not. In the case of fuzzy membership, each gene belongs to a cluster with a membership weight between 0, (definitely excluded), and 1, (definitely included). Clustering algorithms, which permit genes to belong to more than one cluster are typically more applicable to GE since: (i)impact of "noise" is reduced - the assumption is that "noisy" genes are unlikely to belong to any one cluster but are equally likely to be members of several, (ii) this supports the underlying principle that genes, with similar change in expression for a set of samples, are involved in a similar biological function. Typically, gene products are involved in several such biological functions and groups need not be co-active under all conditions. Thus gene groups are fluid and constraining a gene to a single group (hard cluster) is counter-intuitive.

Cluster analysis includes several basic steps [3]. Initially, the data matrix is represented by number, type, dimension and scale of the GE profiles. Some features are set experimentally, others are controllable, (e.g. scaling, imputation, normalisation etc.). An optional step of feature selection or feature extraction may also be carried out. The former refers to selecting, from the original features, a subset, which is most effective for clustering, while the latter refers to transformation of the input features to form a new set that may be more discriminatory in clustering, e.g. through Principal Component Analysis. 
Pattern proximity assessment is needed, usually provided by a "distance" measure between pairs of genes. (Alternatively, "conceptual" measures can be used to characterise similarity of gene profiles e.g. Mean Residue Score of Cheng and Church, (see Section 2)). The next step is to apply a clustering algorithm to determine structure in the dataset. Methods can be broadly categorised according to taxonomy, [3].

Those structures are then described by data abstraction. For GE data, the context is usually direct interpretation by a human, so abstraction should ideally be straightforward (for follow up analysis/experimentation). Required is usually a compact description of each cluster, through a prototype or representative selection of points, such as the centroid. Clusters are valid if they can not reasonably be achieved by chance or as an artefact of the clustering algorithm. Validation requires formal statistical testing, and can be categorised as: (i) Internal, (ii) External or (iii) Relative. The focus here is on proximity measures and clustering algorithms, within the wider analysis context.

\section{Clustering Methods}

Analysis of large GE data-sets is a relatively new task, although pattern recognition of complex data is well-established in a number of fields. Many common generic algorithms have, in consequence, been adopted for GE data, (e.g. Hierarchical [11], SOM's [12], and others), but not all perform well. A good method must deal with noisy high dimensional data, be insensitive to the order of input, have moderate time and space complexity, (i.e. allow increased data load without breakdown or requirement of major changes), require few input parameters, incorporate meta-data knowledge, (an extended range of attributes) and produce results, which are interpretable in the biological context.

\subsection{Pattern Proximity Measures}

The choice of proximity measure, needed to evaluate degree of expression coherence in a group of gene vectors, is as important as choice of clustering algorithm, and is based on data type and context of the clustering. Many clustering algorithms either employ a proximity matrix directly (e.g. hierarchical clustering) or use one to evaluate clusters during execution (e.g. K-Means). Proximity measures are calculated between pairs (e.g. Euclidean distance) or groups of genes (e.g. Mean Residue Error).

Distance functions between two vectors include the so-called Minkowski measures, (Euclidean, Manhattan, Chebyshev, [13]), useful when searching for 
exact matches between two profiles in the dataset. These tend to find globular structures and work well when these are compact and isolated. A drawback is that the largest feature dominates, so measures are sensitive to outliers [3]. However more sophisticated variants, such as Mahalanobis distance, also account for correlations in the dataset and are scale-invariant, [13]. Different distance measures produce clusters of different shape, (e.g Euclidean are spherical, while Mahalanobis' are ellipsoidal). Alternatively, [14] describe an adaptive distance norm (the Gaustafson-Kessel method). Here co-variances are estimated for the data in each cluster, (based on eigenvalue calculations), to obtain structure. Each cluster is then created using a unique distance measure.

Distances based on correlations reflect degree of similarity of changes in expression across samples, for two GE profiles, without regard to scale. For example, if, for a set of samples, gene $X$ is up-regulated, and gene $Y$ is down-regulated, i.e. are correlated, then $X$ and $Y$ would form a cluster. This would clearly not be the case if Minkowski distances were used, since the average absolute distance between the points would be large. Correlation coefficients include both parametric (standard Pearson, cosine), and non-parametric (Spearman's rank and Kendall's $\tau$ ), the latter used when outliers and noise are present, [13]. In general, distance $=1$ - correlation $^{2}$, if sign is unimportant.

As an alternative to measures of distance, "conceptual" measures of similarity can be used. Models are based on constant rows, columns and coherent values, (additive or multiplicative), [10] (Fig. 1). A "good fit" indicates high correlation within a sub-matrix, (thus a possible cluster). These models are common to several clustering algorithms. For example, Cheng and Church [15] and FLOC [16], use the additive model (Fig. 1(C)), to evaluate biclusters obtained by determining the Mean Residue Score. Given a GE matrix $A$, the residue of an element $a_{i j}$ in a sub-matrix $(I, J)$ is given by the difference $r_{i j}=\left(a_{i j}-a_{i J}-a_{I j}+a_{I J}\right)$, where $a_{i j}, a_{i J}, a_{I j}$ and $a_{I J}$ are the sub-matrix value, the row, column and group mean respectively. The "H-score" of the sub-matrix is then the sum of the residues, given by:

$$
H(I, J)=\frac{1}{|I||J|} \sum_{i \in I j \in J}\left(r_{i j}\right)^{2}
$$

A perfect bi-cluster gives a $H$-score equal to zero, (corresponding to "ideal" GE data, with constant additive matrix rows and columns).

The Plaid Model [17] bi-cluster variant builds the GE matrix as a sum of layers, where each layer corresponds to a bi-cluster. Each value $a_{i j}$ is modelled by $a_{i j}=\sum_{k=0}^{K} \theta_{i j k} \rho_{i k} \kappa_{j k}$ where $K$ is the layer (bi-cluster) number, and $\rho_{i k}$ and $\kappa_{j k}$ are binary variables representing membership of row $i$ and column $j$ in layer $k$. The value of an element in the GE matrix can be modelled as a linear 


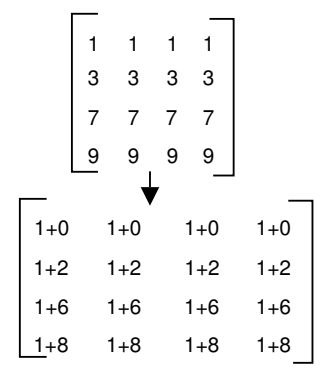

(A) $\mu+\alpha_{i}$

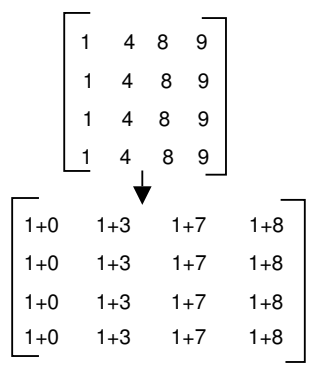

(B) $\mu+\beta_{j}$

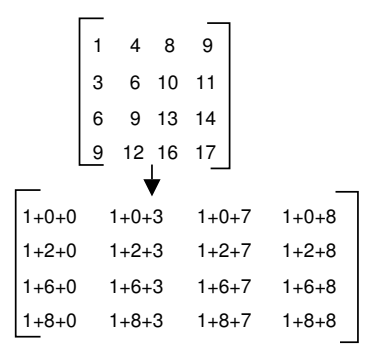

(C) $\mu+\alpha_{i}+\beta_{j}$

Fig. 1. Models for bi-clusters: (A) Bi-cluster with constant rows. Each row is obtained from a typical value $\mu$ and row offset $\alpha_{i}$, (B) Constant columns. Each value is obtained from a typical value $\mu$ and column offset $\beta_{j},(\mathrm{C})$ Additive model. Each value is predicted from $\mu$, and a row and column offset, $\alpha_{i}+\beta_{j}$. Similar model constructs apply for the multiplicative case with $(\mathrm{A}(\mathrm{i})) \mu \times \alpha_{i}$, (B(i)) $\mu \times \beta_{j}$ and (C(i) $\mu \times \alpha_{i} \times \beta_{j}$

function of the contributions of the different bi-clusters to which the row $i$ and the column $j$ belong, (Fig. 2),[17]. For layer $k$, expression level $\theta_{i j k}$ can be estimated using the general additive model, $\theta_{i j k}=\mu_{k}+\alpha_{i k}+\beta_{j k}$, in layer $k$, (Fig. $1(\mathrm{C})$ ).

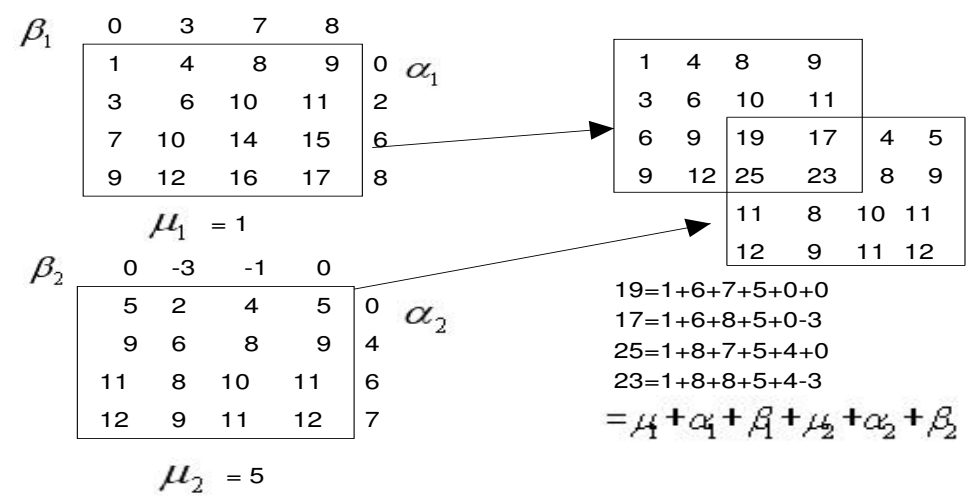

Fig. 2. Plaid Model GE values at overlaps are seen as a linear function of different bi-clusters.

For the Coherent Evolutions model the exact values of $x_{i j}$ are not directly taken into account, but a cluster is evaluated to see if it shows coherent patterns of expression. In simplest model form, each GE value can have three states: up-regulation, down-regulation and no change. Thresholds between states are crucial and additional complexity results from extending model definitions to include further states such as "slightly" up-regulated, "strongly" up-regulated and so on, e.g. SAMBA [18].

Other measures used to evaluate coherency of a group of genes include conditional entropy: $H(C \mid X)=-\int \sum_{j=1}^{m} p\left(c_{j} \mid x\right) \log p\left(c_{j} \mid x\right) p(x) d x$ (the average uncertainty of the random variable $C$ (cluster category), when a random variable $X$ (GE profile) is known). The optimal partition of the GE dataset is obtained 
when this entropy is minimised i.e. a partition where each gene is assigned with a high probability to only one cluster, [19]. This requires the estimation of the a posteriori probabilities $p\left(c_{j} \mid x\right)$, usually by non-parametric methods, as this avoids assumptions on the distribution of the underlying GE data), [19].

Pattern proximity measures described so far make no distinction between timeseries data and those obtained from expressions of two or more phenotypes. Applying similarity measures to time series data is not straightforward. Gene expression time series have non-uniform intervals and are usually very short (4-20 samples while classically even 50 observations is low for statistical inference), further data are not independently, identically distributed data. Similarity in time series should be viewed only in terms of similar patterns in the direction of change across time points, while robust measures must allow for non-uniformity, in addition to scaling and shifting problems, and shape (internal structure of clusters), [20].

Each algorithm described below by definition, relies on some choice of proximity measure and inherits the limitations of that choice.

\subsection{Agglomerative clustering}

All agglomerative techniques naturally form a hierarchical cluster structure in which genes have a crisp membership. Eisen et al. [11] studied GE in the budding yeast, Saccharmyces Cerevisiae, using hierarchical methods which have been popularised due to ease of implementation, visualisation capability and availability. Methods vary with respect to choice of distance metric, decision on cluster merging, (linkage), as well as parameter selection affecting structure and relationship between clusters. Options include: single linkage (cluster separation as distance between two nearest objects), complete linkage (as previously, but between two furthest objects), average linkage (average distance between all pairs), centroid (distance between centroid's of each cluster) and Ward's method, (which minimises ANOVA Sum of Squared Errors between two clusters)[21].

Distance and linkage determine level of sensitivity to noise: Ward's and Complete method are particularly affected, (due to the ANOVA basis and outlier importance respectively, since clustering decisions depend on maximum distance between two genes). Single linkage forces cluster merger, based on minimum distance, regardless of other gene contributions to the cluster, so noisy or outlying values are among the last to be considered. Consequently, the "chaining phenomenon" may arise, [13]. For commonly used Average and Centroid linking this problem is avoided as no special consideration is given 
to outliers and clusters are based on highest density.

Results for agglomerative clustering may be intuitively presented by dendograms but there are $2^{n-1}$ different linear orderings consistent with tree structure, so care is needed in pruning. Dendrogram analysis, based on gene class information from specialised databases is presented in [22], where optimal correlations are obtained between gene classes and used to form clusters from different branch lengths. In [23] authors present an agglomerative technique for which each internal node has at most $N$ children, allowing up to $N$ genes (or clusters) to be directly connected, (extending traditional hierarchical concepts and reducing the effects of noise). Permutation is used to decide on the number of nodes $(\max N)$ to merge, based on a similarity threshold. Heuristically, algorithm complexity is comparable to traditional hierarchical clustering, [23], although the authors also present a "divide and conquer" approach for optimal leaf ordering for small $N$, which has implications of increased time and space complexity.

It should be stated that, such methods can not, in general, compensate for the greedy nature of the traditional algorithm, where mis-clustering at the beginning can not be corrected at a later stage and are magnified as the process continues. Further, [24] and [25] note that hierarchical clustering performance is close to random, despite its popularity and is poorer than other common techniques such as K-means and Self Organising maps (SOM).

\subsection{Partitive Techniques}

Partitive clustering divides data by similarity measure, where typical methods measure distance from a gene vector to a prototype vector representing the cluster, and intra-cluster/inter-cluster distance are respectively maximised and minimised. A major drawback is the need to specify the number of clusters in advance. Table 2 summarises algorithms discussed here.

$K$-means produces crisp clusters with no structural relationship between these, [26]. It deals poorly with noise, since outliers must belong to a cluster and this distort the means. Equally, cluster inclusion is dependent on the cumulative values of genes already present, so order matters. Results are dependent on initial cluster prototype (which varies between clustering attempts); this leads to instability and, frequently, to a local minimum solution. Incremental approaches to refine local minima solutions close towards a global solution, include the Modified Global K-means (MGKM) algorithm [27], which computes $k$-partitions of the data using $k-1$ clusters from previous iterations. A tolerance threshold must be set which determines the number of clusters indirectly, and, as with regular K-means, returns spherical clusters. For the 
six datasets reported [27], the MGKM algorithm showed slight improvement over K-means, but at higher computational time cost.

The prevalence of local minima for $K$-means is linked to initial prototype selection. Genetic algorithms (GAs), as an evolutionary approach, work well for small datasets, (less than 1000 gene vectors and of low dimension), but have prohibitive time constraints for anything larger, so are less desirable for GE analysis. Although GA's find the global optimum, they are sensitive to user defined input parameters and must be fine tuned for each specific problem. Studies which have combined $K$-means and GA include Incremental Genetic K-Means Algorithm (IGKA), [28]. This is a hybrid approach which converges to a global optimum faster than stand alone GA, and without the sensitivity to initialisation prototypes. The fitness function for the GA is based on Total Within Cluster Variance ( $T W C V$ ), while the basis of the algorithm is to cluster centroids incrementally, using a standard similarity measure. The GA method requires the number of output clusters, $K$, to be specified, but is further complicated by inherent GA parameters (mutation probability rate, number of generations, size of the chromosome populations etc.), which influence time taken by the algorithm to converge to a global optimum.

Fuzzy modifications of K-means include Fuzzy C-Means (FCM)[29] and Fuzzy clustering by Local Approximations of MEmberships (FLAME) [30]. In both, genes are assigned a cluster membership degree indicating percentage association with that cluster, but the two algorithms differ in the weighting scheme used to determine gene contribution to the mean. For a given gene, FCM membership value of a set of clusters is proportional to its similarity to cluster mean. The contribution of each gene to the mean of a cluster is weighted, based on its membership grade. Membership values are adjusted iteratively until the variance of the system falls below a threshold. These calculations require the specification of a degree of fuzziness parameter which is problem specific [29]. As with K-Means, clusters are unstable, and considerably influenced by initial parameter values, while $K$, the number of clusters, must be specified a priori. In contrast FLAME requires membership of a cluster, $i$, to be determined by the weighted similarity of the gene to its $K$-nearest neighbours, and their membership of cluster $i$. This density-based approach further reduces noise impact, since genes with a density lower that a predefined threshold are categorised as outliers, and grouped with a dedicated 'outlier' cluster. FLAME produces stable clusters, but the size of the neighbourhood and the weighting scheme used affect $K$ (as above) and clustering achieved. For both FCM and FLAME, genes may have multiple and varied degrees of membership, but interpretation differs. FCM and FLAME use averaging, where each gene contributes to the calculation of a cluster centroid, and its overall membership value set sums to 1, (i.e. gene-cluster probability). Thus strong membership for a given gene does not indicate it to be more typical of the cluster, but rather relative strength of its individual association, 


\begin{tabular}{|r|r|r|r|r|r|r|}
\hline GID & \multicolumn{2}{|c|}{ Cluster 4 } & \multicolumn{2}{c|}{ Cluster 21 } & \multicolumn{2}{c|}{ Cluster 46 } \\
\hline & Centroid Dist. & Mem. & Centroid Dist. & Mem. & Centroid Dist. & Mem. \\
\hline A & 10.691 & 0.002575 & 8.476 & 0.002002 & 3.864 & 0.482479 \\
B & 6.723 & 0.009766 & 3.855 & 0.009341 & 6.33 & 0.007381 \\
C & 6.719 & 0.007653 & 5.29 & 0.00515 & 8.024 & 0.005724 \\
D & 7.725 & 0.007609 & 3.869 & 0.01782 & 6.279 & 0.010249 \\
\hline
\end{tabular}

Table 1

Membership of a gene and distance to cluster centroid, as calculated by Euclidean distance.

$[31]$.

Table 1 illustrates for three clusters. For FCM carried out on published yeast genomic expression data [32], results are available at http://rana.lbl.gov/ FuzzyK/data.html. Membership values for genes B and D are very different for cluster 21, although both are approximately equidistant from the centroid of the cluster. Similarly genes $\mathrm{C}$ and D have comparable membership values for cluster 4, but gene $\mathrm{C}$ is more typical (closer to the centroid) than gene $\mathrm{D}$. With similar centroid distances, membership values for gene B in cluster 21 is smaller than that of gene $\mathrm{A}$ in cluster 46. These anomalies arise from the membership sum constraint, which decreases gene membership in one cluster to increase it in another. Listing genes in a cluster based on membership values is therefore counter-intuitive and does not reflect their compatibility with the cluster, but rather how they are shared between clusters. Similarly for FLAME, as the memberships are weighted relative to the $K$-nearest neighbours, so a low membership value indicates a high degree of cluster sharing among these and not a more typical value og a given cluster. This interpretative flaw was recognised by [33], who developed the possibilistic biclustering algorithm, which removes the sum rule restriction. The authors used spectral clustering principles [34] to create from the original GE matrix, a partition matrix, $Z$, to which possibilistic clustering is applied. The resulting clusters were evaluated using the H-Score, (Eq.1), and improved on traditional techniques. The algorithm requires, inter alia, two specific parameters, namely cutoff membership for (i) gene inclusion and a(ii) sample inclusion in a cluster. In this case, these cutoffs are intuitively reasonable as membership does indicate how typical a gene/sample is to a defined cluster, and not the degree to which it is shared between clusters.

\subsection{Neural Networks}

Neural Networks (NN), loosely based on the biological parallel, can be modelled as a collection of nodes with weighted interconnections. Only numerical vectors are processed, so meta-information can not be included in the clustering procedure. Interconnection weights are adaptively learned i.e. features are 


\begin{tabular}{|c|c|c|c|c|}
\hline & Cluster Mem. & Input & Proximity & Other \\
\hline K-Means & Hard & $\begin{array}{l}\text { Starting Proto- } \\
\text { types, Stopping } \\
\text { Threshold, K }\end{array}$ & $\begin{array}{l}\text { Pairwise } \\
\text { Distance }\end{array}$ & $\begin{array}{l}\text { Very Sensitive } \\
\text { to input parameters } \\
\text { and order of input. }\end{array}$ \\
\hline$M G K M$ & Hard & $\begin{array}{l}\text { Tolerance Thres- } \\
\text { hold }\end{array}$ & $\begin{array}{l}\text { Pairwise } \\
\text { Distance }\end{array}$ & $\begin{array}{l}\text { Not as sensitive to } \\
\text { starting prototypes. } \\
\text { K specified through } \\
\text { tolerance threshold. }\end{array}$ \\
\hline$I G K A$ & Hard & $\begin{array}{l}\mathrm{K} \text {, mutation prob. } \\
\text { generation number, } \\
\text { population size }\end{array}$ & TWCV & $\begin{array}{l}\text { Time taken to } \\
\text { converge to global } \\
\text { influenced by parameters. }\end{array}$ \\
\hline$F C M$ & Fuzzy & $\begin{array}{l}\text { Degree of fuzzi- } \\
\text { ness, Starting } \\
\text { prototypes, Stop } \\
\text { threshold, K }\end{array}$ & $\begin{array}{l}\text { Pairwise } \\
\text { Distance }\end{array}$ & $\begin{array}{l}\text { Careful Interpretation } \\
\text { of membership values. } \\
\text { Sensitive to input para- } \\
\text { metres and order of input. }\end{array}$ \\
\hline$F L A M E$ & Fuzzy & $\begin{array}{l}K_{n n}- \\
\text { number of } \\
\text { neighbours }\end{array}$ & $\begin{array}{l}\text { Pairwise } \\
\text { Distance } \\
\text { to } K_{n n} \\
\text { neighbours }\end{array}$ & $\begin{array}{l}\text { careful interpretation } \\
\text { of membership } \\
\text { values. Output } \\
\text { determined by } K_{n n} \text {. }\end{array}$ \\
\hline $\begin{array}{l}\text { Possibilistic } \\
\text { biclustering }\end{array}$ & Fuzzy & $\begin{array}{l}\text { Cut-off memberships } \\
\text { Max. residue, number of rows } \\
\text { and number of columns }\end{array}$ & H-Score & $\begin{array}{l}\text { Number of biclusers } \\
\text { determined when quality } \\
\text { function peaks by } \\
\text { re-running for different } \\
\text { numbers of eignevalues. }\end{array}$ \\
\hline
\end{tabular}

\section{Table 2}

Summary of Partitive techniques. With the exception of FLAME and Possibilistic biclustering, all find complete global clusters.

selected by appropriate assignment of weights. In particular, Self Organising Maps (SOMs), a type of NN, have proved popular for GE, [12, 35, 36]. A kernel function, that defines the region of influence, (neighbourhood), for an input gene, distinguishes SOM from K-means. Updating the kernel function causes the output node and its neighbours, to track towards the gene vector. The network is trained, (adjusting strengths of interconnections), from a random sample of the dataset. Once training is complete, all genes in the dataset are then applied to the SOM. Cluster members, represented by output node $i$ are the set of genes causing $i$ to 'fire' (hard clustering).

SOMs are robust to noise and outliers, dependent on distance metric and neighbourhood function used. As for K-means, a SOM produces a sub-optimal solution if the initial weights for the interconnections are not chosen properly. Convergence is controlled by problem-specific parameters such as learning rate and neighbourhood function. A particular input pattern can fire different output nodes at different iterations; )while this can be overcome by gradually reducing the learning rate to zero during training, it can result in over-fitting, 
which leads to poor performance for new data). In specifying $K$, based on the number of output nodes, it should be noted that too few output nodes in the SOM gives large within-cluster distance, while too many results in meaningless diffusion.

The Self Organizing Tree Algorithm (SOTA) [37], Dynamically Growing Self Organizing Tree (DGSOT) algorithm [38] and, more recently, Growing Hierarchical Tree SOM (GHTSOM) [39] were developed to combine strengths of NN (i.e. speed, robustness to noise) and hierarchical clustering (i.e. tree structure output, minimum a priori requirement for number of clusters specification and training) to deal with properties of GE data. Here the SOM network is a tree structure, trained by comparing only leaf nodes to input GE profiles (each graph node represents a cluster). SOTA and DGSOT result in a binary and n-tree structure respectively, while in GHTSOM, each node is a triangular SOM (3 neurons, fully connected), each having 3 daughter nodes (also triangular SOMs), Fig. 3. Tree growth strategy determines $K$. At each iteration of SOTA the leaf node with the highest degree of heterogeneity is split into two daughter cells. In the DGSOT case, the correct number of daughters, $\left(n_{d} \geq 2\right)$, is determined dynamically by starting off with two and continually adding one until cluster validation criteria are satisfied. To determine $n_{d}$, a method was proposed [38], based on geometric characteristics of the data (specifically, cluster separation in the minimum spanning tree of the cluster centroids). For this an empirical threshold, $\alpha$, value must be specified; (the authors propose 0.8)). In SOTA and DGSOT, growth of the tree continues until overall heterogeneity crosses a threshold, $\beta$, or until all genes map onto a unique leaf node. The DGSOT method uses average leaf distortion to determine $\beta$ for growth termination. While, for SOTA, this threshold is determined by re-sampling, with system variability defined to be the maximum distance among genes mapped to the same leaf node. By comparing distances between randomized data and those of the real dataset, a confidence interval and distance cut-off are obtained. In GHTSOT, growth occurs if a neuron is activated if a sufficient number of inputs map to it, (i.e. at least 3 or a user defined number, $\beta$ ) which determines the resolution of the system. Growth continues as long as there is one neuron in the system which can grow. The advantage of these methods over most partitive techniques is that $K$ is not pre-determined, but depends indirectly on the threshold, $\beta$, which is data dependant.

SOTA, DGSOT and GHTSOM differ from typical hierarchical clustering algorithms in terms of adaption. This occurs once a gene is mapped to a leaf node, but the neighbourhood of the adaptation is more restrictive than for SOM. DGSOT also overcomes the misclustering problem of the traditional hierarchical algorithm, SOTA and GHTSOM, by specification of another input parameter, $L$ - the immediate ancestor level in the tree of a given node which is growing. DGSOT then distributes all mapped values among the leaves of the subtree rooted at the $L^{\text {th }}$ ancestor. In GHTSOM, new nodes (after growth) 


\begin{tabular}{|l|l|l|l|l|}
\hline & Structure & Proximity & Input & Other \\
\hline SOM & None & Distance & $\begin{array}{l}\text { number of } \\
\text { output neurons, } \\
\text { Learning rate }\end{array}$ & $\begin{array}{l}\text { Careful consideration } \\
\text { of initalisation weights }\end{array}$ \\
\hline SOTA & Binary Tree & Distance & Threshold $\beta$ & \\
\hline DGSOT & N-ary Tree & Distance & $\begin{array}{l}\text { Thresholds } \beta, \alpha \\
\text { and } L \text {. }\end{array}$ & $\begin{array}{l}\text { Corrects for } \\
\text { misclusterings }\end{array}$ \\
\hline GHTSOM & $\begin{array}{l}\text { Each node } \\
\text { triangular SOM, arranged } \\
\text { in Tree structure }\end{array}$ & Distance & $\begin{array}{l}\text { Minimal requirement } \\
- \text { learning rate }\end{array}$ & \\
\hline
\end{tabular}

Table 3

Summary of Neural Network techniques presented.

are trained using only those inputs which caused the parent node to fire. Any neuron, which shows low activity, is deleted, and its parent is blocked from further growth. This has the advantage that inputs mapping to leaf neurons at the top of the hierarchy are usually noise, and clearly distinguishable from relevant biological patterns.

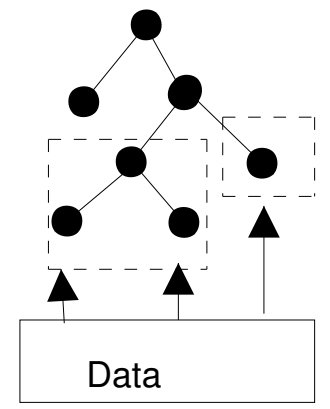

(A) SOTA

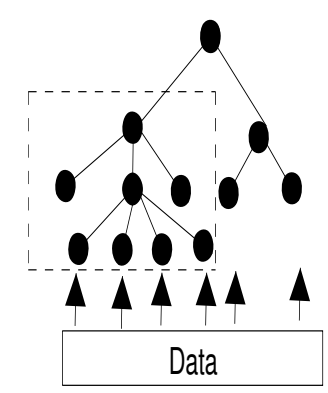

(B) DGSOT

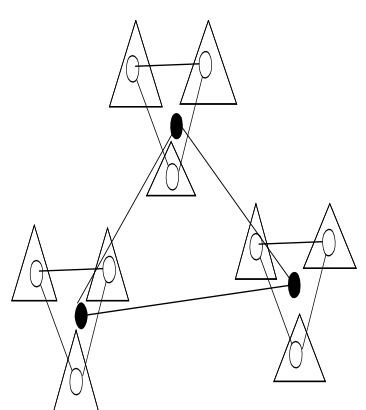

(C) GHTSOM

Fig. 3. Self-Organising tree structures: (A) SOTA. A binary tree structure. Neighbourhood of adaption indicated for (i) node with sibling, (ii) node with no sibling, (B) DGSOT. N-ary tree structure. Neighbourhood of adaption indicated when $L=2$, (C) GHTSOM. Each node represented by triangular SOM. Each layer indicated with line styles, (3 layers shown).

\subsection{Search Based}

Solutions for a criterion function are found by searching the solution space either deterministically or stochastically [3]. The former exhaustive search is of little use for high dimensional GE analysis and, typically, heuristics are used. Simulated Annealing is well-known and has been applied [40] using $T W C V$ to minimise the fitness function, $E$, and, [41], by minimising $H$-Score (Eq. 1). At each stage of the process, gene vectors are randomly chosen and moved to a new random cluster. $E$ is evaluated for each move and the new assignment 
is accepted if $E$ is improved or with a probability of $e^{-\frac{E^{\text {new }}-E^{\text {old }}}{T}}$ otherwise. The "temperature", $T$, controls readiness of the system to accept the poorer situation by chance, enabling the algorithm to avoid local minima. As the search continues, $T$, is gradually reduced according to an annealing schedule, and ultimately achieves the global minimum, where the annealing schedule parameters dictate performance and speed of the search. Choice of initial $T_{i}$ governs convergence time and size of search space, (increased/decreased in the case of high/low $T$ respectively). Similarly for search termination, (final effective $T_{F}$ ). The user must specify the rate at which $T$ approaches $T_{F}$, which must be slow enough to guarantee a global minimum, as well as the number of swaps of gene vectors between clusters allowed in an iteration.

To determine $K$, a randomisation procedure is used, [40], to determine cut-off threshold for the distance, $D$, between two gene vectors in a single cluster. It is also necessary to determine $P$, the probability of accepting false positives, (e.g. $P=0.05$ ). Simulated annealing is then applied for different numbers of clusters, until the weighted average fraction of incorrect gene vector pairs reaches the $P$-value.

The algorithm of Cheng and Church [15] (adapted from Hartigan [42]) obtains H-scores, (Eq. 1, Fig. 1) [10]) of the sub-matrices of the GE matrix. This method is initialised for the entire GE matrix and considers a sub-matrix to be a bi-cluster if $H(I, J)<\delta$ for some $\delta \geq 0$, (user defined). Each row and column of the original matrix is thus tested for deletion. Once a sub-matrix is determined to be a bi-cluster, its values are "masked" with random numbers in the initial GE matrix. Masking bi-clusters prevents the algorithm from repeatedly finding the same sub-matrices, but there is a substantial risk that this replacement will interfere with the discovery of future bi-clusters, [16]. To overcome this problem of random interference, Flexible Overlapped biClustering (FLOC) was developed - a generalised model of Cheng and Church incorporating null values, [16]. FLOC constrains the clusters to both a low mean residue score and a minimum occupancy threshold of $\alpha, 0 \leq \alpha \leq 1$ (user defined). Note: this method does not require pre-processing for imputation of missing values. Both, these bi-clustering algorithms find coherent groups (Section 2.1) in the data and permit overlapping.

The Plaid Model [17] (Section 2.1) assumes that bi-clusters can be generated using a statistical model and aims to identify the parameter distribution that best fit the available data, by minimising the error sum of squares for the $k^{\text {th }}$ bi-cluster assuming that $k-1$ bi-clusters have already been identified. Explicitly, it seeks to minimise for the whole matrix: $Q=\frac{1}{2} \sum_{i=1}^{n} \sum_{j=1}^{m}\left(Z_{i j}-\right.$ $\left.\theta_{i j k} \rho_{i k} \kappa_{j k}\right)^{2}$, where $Z_{i j}$ is the residual after deducting $k-1$ previous layers, 


\begin{tabular}{|l|l|l|l|l|}
\hline & Proximity & $\begin{array}{l}\text { Deterministic/ } \\
\text { Stochastic }\end{array}$ & Clusters & Other \\
\hline$S A$ & $\begin{array}{l}\text { Depends on } \\
\text { application }\end{array}$ & Stochastic & $\begin{array}{l}\text { Depends on } \\
\text { application }\end{array}$ & $\begin{array}{l}\text { Specification of } \\
\text { Annealing Schedule }\end{array}$ \\
\hline AC & Additive Model & Deterministic & $\begin{array}{l}\text { Overlapping, partial } \\
\text { bi-clusters }\end{array}$ & $\begin{array}{l}\delta, \\
\text { random interference }\end{array}$ \\
\hline FLOC & Additive Model & Deterministic & $\begin{array}{l}\text { Overlapping, partial } \\
\text { bi-clusters }\end{array}$ & $\begin{array}{l}\alpha \text { and } \delta \\
\text { to specify. Overcomes } \\
\text { random interference, } \\
\text { allows missing values. }\end{array}$ \\
\hline Plaid & Additive Model & Deterministic & $\begin{array}{l}\text { Overlapping, partial } \\
\text { bi-clusters }\end{array}$ & $\begin{array}{l}\text { Values seen as sum of } \\
\text { contributions to bi-clusters }\end{array}$ \\
\hline
\end{tabular}

Table 4

Summary of search based techniques presented.

$\left(Z_{i j}=a_{i j}-\sum_{k=0}^{K-1} \theta_{i j k} \rho_{i k} \kappa_{j k}\right)$. Parameters $\theta_{i j k}, \rho_{i k}$ and $\kappa_{j k}$ are estimated for each layer and for each value in the matrix, and are updated iteratively, providing refined estimates of $\mu_{k}, \alpha_{i k}$ and $\beta_{j k}$, (Fig: $1(\mathrm{C})$ ) and $\rho_{i k}$ and $\kappa_{j k}$ to minimise $Q,[17]$.

The importance of a layer is defined by $\delta_{k}^{2}=\sum_{i=1}^{n} \sum_{j=1}^{p} \rho_{i k} \kappa_{j k} \theta_{i j k}^{2}$. To evaluate the significance of the residual matrix, $Z$ is randomly permuted and tested for importance. If $\delta_{k}^{2}$ is significantly better than $\delta_{\text {random }}^{2}, k$ is reported as a bi-cluster. The algorithm stops when the residual matrix $Z$ retains only noise, with the advantage that the user does not need to specify the number of clusters beforehand.

\subsection{Graph Theoretic Methods}

Graph theoretic approaches have recently gained ground in analysing large complex datasets. The Cluster Affinity Search Technique (CAST), [43], models data as an undirected graph, $G=(V, E)$, where $\{V, E\}$ is the set of $\{$ vertices, edges $\}$ representing $\{$ genes, similarexpression $\}$. The model assumes that there is an ideal clique graph, (a disjoint union of complete sub-graphs), $H=(U, E)$, which represents the ideal input gene expression dataset, while data to be clustered is a "contamination" of the ideal graph $H$ by random errors. In a clique graph each clique represents a cluster. For a pair of genes in $G$, the model assumes that an edge/non-edge was assigned incorrectly, with a probability of $\alpha$. The true clustering of $G$ is assumed to be that which requires fewer edge changes to generate $H$. CAST uses an affinity (similarity) measure, either binary or real valued, to assign a vertex to a cluster. Affinity to a cluster must be above a threshold, $t$ (user defined which determines size and number of clusters). The affinity of a vertex $v$ to a cluster, is the 
sum of affinities over all objects currently in the cluster, so $v$ has high affinity with $i$ if affinity $(x)>t|i|$, and low affinity otherwise. The CAST algorithm alternates between adding high affinity elements and removing low affinity elements, finding clusters one at a time. The result is dependent on the order of input as once initial cluster structure is obtained, a vertex $v$ is moved to that cluster for which it has a higher affinity value.

CLICK [44], builds on the work of [45], which uses a probabilistic model for edge weighting. Pairwise similarity measures between genes are assumed to be normally distributed: between 'mates' $\left(N\left(\mu_{T}, \sigma_{T}^{2}\right)\right)$, and between 'nonmates' $\left(N\left(\mu_{F}, \sigma_{F}^{2}\right)\right)$, where $\mu_{T}>\mu_{F}$. These parameters can be estimated via Expectation Maximisation methods [46]. The weight of an edge is derived from the similarity measure between the two gene vectors, and reflects the probability that $i(\in V)$ and $j(\in V)$ are mates, specifically that: $w_{i j}=\log \frac{p_{\text {mates }} \sigma_{F}}{\left(1-p_{\text {mates }}\right) \sigma_{T}}+\frac{\left(S_{i j}-\mu_{F}\right)^{2}}{2 \sigma_{F}^{2}}-\frac{\left(S_{i j}-\mu_{T}\right)^{2}}{2 \sigma_{T}^{2}}$. Edges with weights below a user defined non-negative threshold are omitted from the graph. The graph is partitioned using a minimum weight cut algorithm [45].

The Statistical Algorithmic Method for Bi-cluster Analysis (SAMBA) method finds bi-clusters based on the coherent evolution model (Section 2.1) [18]. Firstly, the gene expression matrix is modelled as a bipartite graph, $G=$ $(U, V, E)$, where $U$ is the set of sample vertices, $U \cap V=\varnothing$ and an edge $(u, v)$ only exists between $v \in V$ and $u \in U$ iff there is a significant change in expression level of gene $v$, w.r.t. to its normal level, in sample $u$. Key to SAMBA is the scoring scheme for a bi-cluster, corresponding to its statistical significance, where a weight is assigned to a given edge, $(u, v)$, based on the log-likelihood of getting that weight by chance [18], $\left(\log \frac{P_{c}}{P_{(u, v)}}>0\right.$ for edges and, $\log \frac{\left(1-P_{c}\right)}{\left(1-P_{(u, v)}\right)}<0$ for non-edges). The probability $P_{(u, v)}$ is the fraction of random bipartite graphs, with degree sequence identical to $G$, that contain edge $(u, v)$ (and can be estimated using Monte-Carlo methods). $P_{c}$ is a constant probability $>\max _{(u, v) \in U x V} P_{(u, v)}$. Assigning these weights to the edges and non-edges in the graph, the statistical significance of a subgraph $H$ can be calculated, and the $K$ heaviest (largest weight) sub-graphs for each vertex in $G$ found. The authors, [18] present two ways to calculate the weight of the resulting sub-graph. In the simpler model, bi-clusters, which reflect changes relative to normal expression level, without considering direction of change are sought. The second model, focuses on consistent bi-cliques, targeting those samples which have the same or opposite effect on each of the genes. 


\begin{tabular}{|l|l|l|l|l|}
\hline & Mode & Proximity & Search & Other \\
\hline CAST & One Mode & Similarity & $\begin{array}{l}\text { Clique } \\
\text { Graph }\end{array}$ & $\begin{array}{l}\text { Parameters } \alpha \text { and } t \text {. Finds global, } \\
\text { complete, crisp clusters. }\end{array}$ \\
\hline CLICK & One Mode & $\begin{array}{l}\text { Distribution } \\
\text { based on } \\
\text { distance }\end{array}$ & $\begin{array}{l}\text { Minimum } \\
\text { weight cut }\end{array}$ & $\begin{array}{l}\text { Stat. Sig. of clusters. EM to } \\
\text { estimate parameters. Finds } \\
\text { global, partial, crisp clusters. }\end{array}$ \\
\hline SAMBA & Bi-Partite & Probability & $\begin{array}{l}\text { Heuristic } \\
\text { search of } \\
\text { neighbours }\end{array}$ & $\begin{array}{l}\text { Stat. sig. of clusters. Input } \\
P_{c} \text { difficult to define. Finds } \\
\text { partial overlapping bi-clusters. }\end{array}$ \\
\hline
\end{tabular}

Table 5

Summary of performance criterion of Graph theoretic methods presented.

\section{Discussion}

Despite shortcomings, application of clustering methods to GE data has proven to be of immense value, providing insight on cell regulation, as well as on disease characterisation. Nevertheless, not all clustering methods are equally valuable for high dimensional GE data. Recognition that well-known, simple clustering techniques, such as K-Means and Hierarchical clustering, do not capture complex local structure, has led to investigation of other options. In particular, bi-clustering has gained considerable recent popularity. Indications to date are that these methods provide increased sensitivity at local structure level in discovery of meaningful biological patterns.

An inherent problem with exploratory clustering is ab initio knowledge of $K$, the number of clusters. Consequently, those methods for GE analysis which do not need $K$ specified ab initio have an advantage. Most algorithms seek empirically to determine this at run time, but derive complicated thresholds that may not make sense in the context of gene expression data. There is a risk that determination of these thresholds is not a one step process but requires testing and validation of clusters produced. While space limits a comprehensive survey of robust cluster validation and evaluation methods here, their importance is clear: (see [47] for a comprehensive review). A discipline of informationdriven clustering is emerging, which integrates cluster and meta-information, $[48,49,50,51,52]$. These provide a basis for validation, independent of the current problem and simplify interpretation of clustering results.

\section{Conclusion}

Cluster analysis applied to GE data aims to highlight meaningful patterns for gene co-regulation. The evidence suggests that, while commonly applied, agglomerative and partitive techniques are insufficiently powerful given the high dimensionality and nature of the data. While further testing on non-standard 
and diverse data sets is required, comparative assessment and numerical evidence, to date, supports the view that bi-clustering methods, although computationally expensive, offer better interpretation in terms of data features and local structure. While the limitations of commonly-used algorithms are well documented in the literature, adoption by the bioinformatic community of new (and hybrid) techniques developed specifically for GE analysis has been slow , mainly due to the increased algorithmic complexity required. This would be catalysed by more transparent guidelines and increased availability in specialised software and public dataset repositories.

\section{References}

[1] M. H. Asyali, D. Colak, O. Demirkaya, and M. S. Inan. Gene expression profile classification: A review. Curr. Bioinformatics, 1(1):55-73, 2006.

[2] T. D. Pham, C. Wells, and D. I. Crane. Analysis of microarray gene expression data. Curr. Bioinformatics, 1(1):37-53, 2006.

[3] A. K. Jain, M. N. Murty, and P. J. Flynn. Data clustering: A review. ACM CSUR, 31(3):264-323, 1999.

[4] S. O. Zakharkin, K. Kim, T. Mehta, L. Chen, S. Barnes, K. E. Scheirer, R. S. Parrish, D. B. Allison, and G. P. Page. Sources of variation in affymetrix microarray experiments. BMC bioinformatics, 6:214, Aug 29 2005.

[5] O. Troyanskaya, M. Cantor, G. Sherlock, P. Brown, T. Hastie, R. Tibshirani, D. Botstein, and R. B. Altman. Missing value estimation methods for dna microarrays. Bioinformatics, 17(6):520-525, Jun 2001.

[6] X. Liu, G. Cheng, and J. X. Wu. Analyzing outliers cautiously. IEEE Trans. Knowl. Data Eng., 14(2):432-437, 2002.

[7] G. W. Milligan and M. C. Cooper. An examination of procedures for determining the number of clusters in a dataset. Psychometrika, 50:159179, 1985.

[8] J. Fridlyand and S. Dudoit. Applications of resampling methods to estimate the number of clusters and to improve the accuracy of a clustering method. Technical Report 600, Department of Statistics, University of California, Berkeley, 2001.

[9] A. Ben-Dor, B. Chor, R. Karp, and Z. Yakhini. Discovering local structure in gene expression data: the order-preserving submatrix problem. $J$. Comput. Biol., 10(3-4):373-384, 2003.

[10] S. C. Madeira and A. L. Oliveira. Biclustering algorithms for biological data analysis: a survey. IEEE/ACM Trans. Comput. Biol. Bioinform., 1(1):24-45, 2004.

[11] M. B. Eisen, P. T. Spellman, P. O. Brown, and D. Botstein. Cluster analysis and display of genome-wide expression patterns. Proc. Natl. Acad. Sci USA, 95(25):14863-14868, Dec 81998. 
[12] T. Kohonen. The self-organizing map. Proceedings of the IEEE, 78(9):1464-1480, 1990.

[13] C. Romesburg. Cluster Analysis for Researchers. Lulu Press, Morrisville, 2004.

[14] D. W. Kim, K. H. Lee, and D. Lee. Detecting clusters of different geometrical shapes in microarray gene expression data. Bioinformatics, 21(9):1927-1934, May 12005.

[15] Y. Cheng and G. M. Church. Biclustering of expression data. ISMB '00, 8:93-103, 2000.

[16] J. Yang, H. Wang, W. Wang, and P. Yu. Enhanced biclustering on expression data. BIBE' '03, page 321, 2003.

[17] L. Lazzeroni and A. B. Owen. Plaid models for gene expression data. Statistica Sinica, 12(1):61-86, 2000.

[18] A. Tanay, R. Sharan, and R. Shamir. Discovering statistically significant biclusters in gene expression data. Bioinformatics, 18 Suppl 1:S136-44, 2002.

[19] H. Li, K. Zhang, and T. Jiang. Minimum entropy clustering and applications to gene expression analysis. Proc. IEEE CSB., pages 142-151, 2004.

[20] C. Moller-Levet, K. H. Cho, H. Yin, and O. Wolkenhauer. Clustering of gene expression time-series data. Technical report, 2003.

[21] A. Sturn. Cluster analysis for large scale gene expression studies, 2001.

[22] P. Toronen. Selection of informative clusters from hierarchical cluster tree with gene classes. BMC bioinformatics, 5:32, Mar 252004.

[23] Z. Bar-Joseph, E. D. Demaine, D. K. Gifford, N. Srebro, A. M. Hamel, and T. S. Jaakkola. K-ary clustering with optimal leaf ordering for gene expression data. Bioinformatics, 19(9):1070-1078, Jun 122003.

[24] K. Y. Yeung, D. R. Haynor, and W. L. Ruzzo. Validating clustering for gene expression data. Bioinformatics, 17(4):309-318, Apr 2001.

[25] F. D. Gibbons and F. P. Roth. Judging the quality of gene expressionbased clustering methods using gene annotation. Genome Res., 12(10):1574-1581, Oct 2002.

[26] J. B. MacQueen. Some methods for classification and analysis of multivariate observations. In L. M. Le Cam and J. Neyman, editors, Proc. Fifth Berkeley Symp. Math. Stat. Prob., pages 281-297. University of California Press, 1967.

[27] Adil M. Bagirov and Karim Mardaneh. Modified global k-means algorithm for clustering in gene expression data sets. In WISB '06, pages 23-28, Darlinghurst, Australia, 2006. Australian Computer Society, Inc.

[28] Y. Lu, S. Lu, F. Fotouhi, Y. Deng, and S. J. Brown. Incremental genetic $\mathrm{k}$-means algorithm and its application in gene expression data analysis. BMC bioinformatics, 5:172, Oct 282004.

[29] D. Dembele and P. Kastner. Fuzzy c-means method for clustering microarray data. Bioinformatics, 19(8):973-980, May 222003.

[30] L. Fu and E. Medico. Flame, a novel fuzzy clustering method for the 
analysis of dna microarray data. BMC bioinformatics, 8:3, Jan 42007.

[31] R. Krishnapuram and J. M. Keller. A possibilistic approach to clustering. IEEE TFS, 1(2):98-110, 1993.

[32] A. P. Gasch and M. B. Eisen. Exploring the conditional coregulation of yeast gene expression through fuzzy k-means clustering. Genome biology, 3(11):RESEARCH0059, Oct 102002.

[33] C. Cano, L. Adarve, J. Lopez, and A. Blanco. Possibilistic approach for biclustering microarray data. Comp. Biol. Med., 37(10):1426-1436, Oct 2007.

[34] Y. Kluger, R. Basri, J. T. Chang, and M. Gerstein. Spectral biclustering of microarray data: coclustering genes and conditions. Genome Res., 13(4):703-716, Apr 2003.

[35] P. Tamayo, D. Slonim, J. Mesirov, Q. Zhu, S. Kitareewan, E. Dmitrovsky, E. S. Lander, and T. R. Golub. Interpreting patterns of gene expression with self-organizing maps: methods and application to hematopoietic differentiation. Proc. Natl. Acad. Sci. USA, 96(6):2907-2912, Mar 161999.

[36] T. R. Golub, D. K. Slonim, P. Tamayo, C. Huard, M. Gaasenbeek, J. P. Mesirov, H. Coller, M. L. Loh, J. R. Downing, M. A. Caligiuri, C. D. Bloomfield, and E. S. Lander. Molecular classification of cancer: class discovery and class prediction by gene expression monitoring. Science, 286(5439):531-537, Oct 151999.

[37] J. Herrero, A. Valencia, and J. Dopazo. A hierarchical unsupervised growing neural network for clustering gene expression patterns. Bioinformatics, 17(2):126-136, Feb 2001.

[38] F. Luo, L. Khan, F. Bastani, I. L. Yen, and J. Zhou. A dynamically growing self-organizing tree (dgsot) for hierarchical clustering gene expression profiles. Bioinformatics, 20(16):2605-2617, Nov 12004.

[39] A. Forti and G. L. Foresti. Growing hierarchical tree som: an unsupervised neural network with dynamic topology. Neural networks, 19(10):15681580, Dec 2006.

[40] A. V. Lukashin and R. Fuchs. Analysis of temporal gene expression profiles: clustering by simulated annealing and determining the optimal number of clusters. Bioinformatics, 17(5):405-414, May 2001.

[41] K. Bryan, P. Cunningham, and N. Bolshakova. Application of simulated annealing to the biclustering of gene expression data. IEEE T-ITB, 10(3):519-525, Jul 2006.

[42] J. A. Hartigan. Direct clustering of a data matrix. JASA, 67(337):123$129,1972$.

[43] A. Ben-Dor, R. Shamir, and Z. Yakhini. Clustering gene expression patterns. J. Comput. Biol., 6(3-4):281-297, Fall-Winter 1999.

[44] R. Sharan and R. Shamir. Click: a clustering algorithm with applications to gene expression analysis. ISMB '00, 8:307-316, 2000.

[45] E. Hartuv, A. O. Schmitt, J. Lange, S. Meier-Ewert, H. Lehrach, and R. Shamir. An algorithm for clustering cdna fingerprints. Genomics, 66(3):249-256, Jun 152000. 
[46] A. P. Dempster, N. M. Laird, and D. B. Rubin. Maximum likelihood from incomplete data via the em algorithm. J Royal Statistical Soc B (Methodological), 39(1):1-38, 1977.

[47] J. Handl, J. Knowles, and D. B. Kell. Computational cluster validation in post-genomic data analysis. Bioinformatics, 21(15):3201-3212, Aug 1 2005.

[48] G. Gamberoni, S. Storari, and S. Volinia. Finding biological process modifications in cancer tissues by mining gene expression correlations. BMC Bioinformatics, 7(6):8, Jan 92006.

[49] R. Kustra and A. Zagdanski. Incorporating gene ontology in clustering gene expression data. CBMS, 0:555-563, 2006.

[50] J. Kasturi and R. Acharya. Clustering of diverse genomic data using information fusion. Bioinformatics, 21(4):423-429, 2005.

[51] J.K. Choi, J.Y. Choi, D.G. Kim, D.W. Choi, B.Y. Kim, K.H. Lee, Y.I. Yeom, H.S. Yoo, O.J. Yoo, and S. Kim. Integrative analysis of multiple gene expression profiles applied to liver cancer study. FEBS Letters, 565(1-3):93-100, 2004.

[52] J. Liu, W. Wang, and J. Yang. Gene ontology friendly biclustering of expression profiles. In CSB '04, pages 436-447, Washington, DC, USA, 2004. IEEE Computer Society. 